\title{
Performance of a Non-Coherent Massive SIMO M-DPSK System
}

\author{
Victor Monzon Baeza ${ }^{1}$, Ana Garcia Armada ${ }^{1}$, Mohammed El-Hajjar ${ }^{2}$ and Lajos Hanzo ${ }^{2}$ \\ ${ }^{1}$ University Carlos III of Madrid, Leganes (Madrid), Spain \\ ${ }^{2}$ Department of ECS, University of Southampton, SO17 1BJ, United Kingdom \\ vmonzon@tsc.uc3m.es, agarciadtsc.uc3m.es, meh@ecs.soton.ac.uk, lh@ecs.soton.ac.uk
}

\begin{abstract}
In this paper, we analyze the effect of time-varying channels on the performance of a non-coherent massive singleinput multiple-output (SIMO) uplink system based on $M$-ary Differential Phase Shift Keying (M-DPSK), when amalgamated with bit-interleaved coded modulation relying on iterative decoding (BICM-ID). Additionally, we study the number of receive antennas $(R)$ required in hostile time-varying channels for attaining a similar performance to that achieved for stationary channels. Furthermore, we analyze the maximum achievable rate (MAR) in conjunction with the specific modulation and coding schemes considered. The analysis is based on extrinsic information transfer (EXIT) charts parametrized by $R$, which varies with the signal to interference-plus-noise ratio (SINR). Our numerical results show that the system is robust to the effects of the channel's temporal correlation and that the simulationbased MAR closely matches the semi-analytical achievable rate obtained using EXIT charts. Hence, our system can be applied to scenarios associated with the short coherence time of high-speed railway systems, for example.
\end{abstract}

\section{INTRODUCTION}

Recently, wireless networks have experienced an increasing demand for higher data rates and traffic volumes due to the increasing popularity of mobile multimedia services. Hence, massive multiple input-multiple output (m-MIMO) systems have been proposed as a promising solution for providing a substantial increase in the attainable network capacity [1]. The m-MIMO systems employ a high number of antennas at the Base Station (BS). Hence, m-MIMO systems hold a substantial promise for future wireless communication systems [2].

In general, when utilizing the conventional coherent detection, m-MIMO techniques require the knowledge of the channel state information (CSI) at the BS, where a large number of channels have to be estimated. The CSI may be obtained using pilot signals transmitted from each user to the $\mathrm{BS}$, assuming reciprocity in the radio link when employing time division duplexing (TDD). Due to the fact that the pilot signals used in the adjacent cells are not completely orthogonal, the performance of the m-MIMO systems is degraded by the widely-recognized pilot contamination [3].

Therefore, non-coherent (NC) detection consitutes an attractive design alternative that eliminates the need for any channel estimation. In this context, the authors of [4] proposed an Amplitude Shift Keying (ASK) system for NC detection dispensing with the CSI, which exploits the impact of random coding for reducing the required number of antennas. However, to guarantee a good performance, the design proposed in
[4] requires an impractically large number of receive antennas at the BS.

In our previous work [5], we proposed a novel scheme relying on $M$-ary Differential Phase Shift Keying (M-DPSK), amalgamated with a powerful coding scheme based on the principle of Bit-Interleaved Coded Modulation and Iterative Decoding (BICM-ID) [6] for non-coherent massive single input multiple output (m-SIMO) systems. We showed that the employment of BICM-ID considerably reduces the number of receive antennas required for attaining a specific target performance compared to [4]. In [5] we considered a static channel, where the differential detection requires the channel to be static for two consecutive time slots, which is an assumption that can be shown to hold in most of the wireless applications that we envisage today.

In terms of the carrier frequency, the use of millimeterwave (mm-wave) frequencies is becoming increasingly more popular in conjunction with m-MIMO systems [7], which is motivated both the compact nature of the large-scale antennas as well as by the promise of abundant spectrum above 10 GHz. Additionally, communication in the Terahertz band is considered for satisfying the high traffic demands [8]. Hence, the use of these high frequencies as well as the support of highspeed vehicles implies that the channel is time-variant and the above assumption does not necessarily hold. This motivates the analysis of our proposed scheme under time varying channels.

In this paper, we consider links characterized by short coherence times in order to analyze our non-coherent system in fast-fading channels. Additionally, we exploit the energyefficiency of m-SIMOs to operate at low SNRs. The novel contribution of this work can be summarized as follows:

- We extend the analysis of our non-coherent m-SIMO system in [5] to include the impact of time-varying channels on the required number of antennas and to the analysis of the maximum achievable rate (MAR).

- We demonstrate that the proposed scheme is suitable for channels exhibiting short coherence times. Our analysis is based on a novel metric reflecting the channel's variability that we further illustrate with the aid of practical channels.

The rest of the paper is organized as follows. The proposed system model is introduced in Section II. Then the required number of antennas is analyzed in Section III, followed by the analysis of the maximum achievable rate in Section IV. 
Finally, we draw our conclusions in Section V.

\section{Proposed System Model}

In this paper we consider an uplink scenario, where $P$ users or Mobile Stations (MSs) communicate with a BS equipped with $R$ receive antennas (RA). Specifically, we consider $P=2$ users, as shown in Fig. 1.

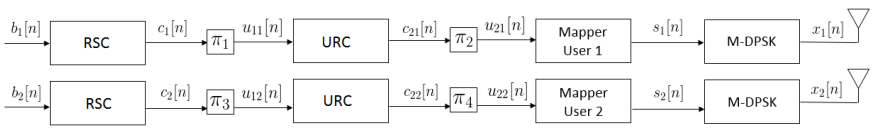

Fig. 1. Transmitter of a Non Coherent m-SIMO system for $P=2$ users.

We assume that user $i$ transmits the signal $x_{i}[n]$ at time instant $n$, where the signals generated by the two users are clustered into the vector $\boldsymbol{x}$ of size $(P \times 1)$. Each $x_{i}[n]$ is a differentially encoded version of $s_{i}[n]$ formulated as

$$
x_{i}[n]=s_{i}[n] x_{i}[n-1], n>1 .
$$

A block of bits $b_{i}[n]$ are encoded by a recursive systematic convolutional (RSC) code as an outer encoder and then randomly interleaved by the bit interleaver $\pi_{1}$ to be encoded by a unity-rate code (URC) encoder as an inner encoder, giving $c_{2 i}[n]$. This bit stream is interleaved again by the second interleaver $\pi_{2}$, providing the sequence $u_{2 i}[n]$. Then, these bits are mapped to symbols $s_{i}[n]$ which belong to an $M$-ary constellation $\mathfrak{M}_{i}=\left\{s_{i, m}, m=0, \ldots, M-1\right\}$, where $\left|s_{i, m}[n]\right|=1$ and $M$ is the order of the constellation for each user. The $x_{i}[0]$ is known both at the transmitter and receiver.

The m-MIMO wireless channel is modeled using a matrix $\boldsymbol{H}$ of size $(R \times P)$, whose elements $h_{j, i} \sim C N(0,1)$ represent the propagation between user $i$ and the $j$-th antenna at the BS. The channel matrix accounts for Rayleigh fading with zero mean and variance 1, where temporal correlation will be introduced as explained later. Each of the antennas at the BS receives the vector $\boldsymbol{y}_{j}[n]$ at time instant $n$, which is obtained as $\boldsymbol{y}=\boldsymbol{H} \boldsymbol{x}+$ $\mathbf{v}^{1}$. The AWGN vector $\mathbf{v}$ consists of $(R \times 1)$ elements, where $v_{j} \sim C N\left(0, \sigma^{2}\right)$. The reference SNR is evaluated as $\rho=\frac{P}{\sigma^{2}}$. Since the power of each individual user is normalized, the total power is $\sum_{i=1}^{P}\left|s_{i, m}[n]\right|^{2}=P$. Hence, $\rho$ depends on the number of users $P$.

At the receiver characterized in Fig. 2, we assume that $h_{j i}[n]=h_{j i}[n-1]=h_{j i}, j=1, \ldots, R, i=1, \ldots, P .^{2}$ Then, the phase difference is non-coherently detected for these two symbols for each receive antenna. The resultant received symbol $z[n]$ is

$$
z[n]=\frac{1}{R} \sum_{j=1}^{R} y_{j}^{*}[n-1] y_{j}[n] .
$$

The symbols $z[n]$ collect both the information and interference arriving from all users. As shown in Fig. 2, these symbols are converted to their log-likelihood ratio (LLR) representation,

\footnotetext{
${ }^{1}$ In the following, we will drop the time dependency $n$ to simplify our notation.

${ }^{2}$ We will analyze later the effect of this assumption when considering timevarying channels.
}

$L_{M}(z[n])=\left\{L\left(u_{1}\right), L\left(u_{2}\right)\right\}$ by the soft-demapper block in order to decide on the joint received symbol $\varsigma[n]$, defined as

$$
\varsigma[n]=\sum_{i=1}^{P} s_{i}[n] .
$$

Then, expanding (3) which is not shown here for the sake of conciseness, we have

$$
z[n] \stackrel{R \rightarrow \infty}{=} \varsigma[n]+i[n],
$$

where $i[n]$ are the noise terms and the interference imposed by all the antennas and users. The power of the interference $I=E\left\{|i[n]|^{2}\right\}$ depends on $R$ and $P$ as follows

$$
I=\frac{P^{2}+2 \sigma^{2} P+\sigma^{4}}{R} \text {. }
$$

In order to obtain the LLR for each possible value of $\varsigma_{m}$, the soft demapper uses the metric in the log-domain given by [5]

$$
\gamma_{m}(z[n])=-\frac{\left|z[n]-\varsigma_{m}\right|^{2}}{2 I} .
$$

A value of $\gamma_{m}(z[n])$ for each $\varsigma_{m}$ is obtained. The highest $\gamma_{m}(z[n])$ corresponds to the most likely $\varsigma$. Then, this highest value of $\gamma_{m}(z[n])$, denoted as $L_{M}(z[n])$, is passed to the splitter shown in Fig. 2 . The splitter separates the soft values of each user $L\left(u_{P}\right)$ from the $(P \cdot M)$ values in $L_{M}(z[n])$ according to the mapping performed at the transmitter. This separation is merely a serial-to-parallel conversion, which does not involve any mathematical operations.

Afterwards, the decoding process is performed independently for each user $i$. The URC decoder processes the information provided by the demapper, deinterleaved by $\pi_{2}$, in conjunction with the a priori information $L_{i n, a}\left(u_{1, i}\right)$ passed back to it from the RSC decoder, in order to generate the extrinsic LLRs $L_{i n, e}\left(u_{1 i}\right)$. Then, the LLRs $L_{i n, e}\left(u_{1, i}\right)$ are deinterleaved by a soft-bit deinterleaver $\pi_{1}$, as seen in Fig. 2. Next, the soft-bit values $L_{o, a}\left(c_{i}\right)$ are passed to the RSC decoder which uses the classic BCJR algorithm [9] in order to compute the extrinsic LLRs $L_{o, e}\left(c_{i}\right)$. This procedure is repeated iteratively. During the last iteration, only the a posteriori LLR values, $L_{p}\left(b_{i}\right)$, of the original uncoded systematic information bits are required, which are passed to a hard decision decoder in order to determine the estimated transmitted source bits $\hat{b}_{i}$.

\section{A. EEP Constellation and Coding Scheme}

We will aim for providing the same Bit Error Ratio (BER) performance for all users, which we denote as Equal Error Protection (EEP) design in [5]. The constellation $\mathfrak{M}_{i}$ for user $i$ is defined as

$$
\mathfrak{M}_{i}^{E E P}=\left\{\frac{2 \pi[(m+1) P-1+i]}{P M}, \begin{array}{c}
m=0, \ldots, M-1 \\
i=1, \ldots, P
\end{array}\right\} .
$$

We will use the powerful extrinsic information transfer (EXIT) chart tool for analysing the constellation design and for finding the best coding scheme for our system based on the number of antennas. Based on the interference analysis in 


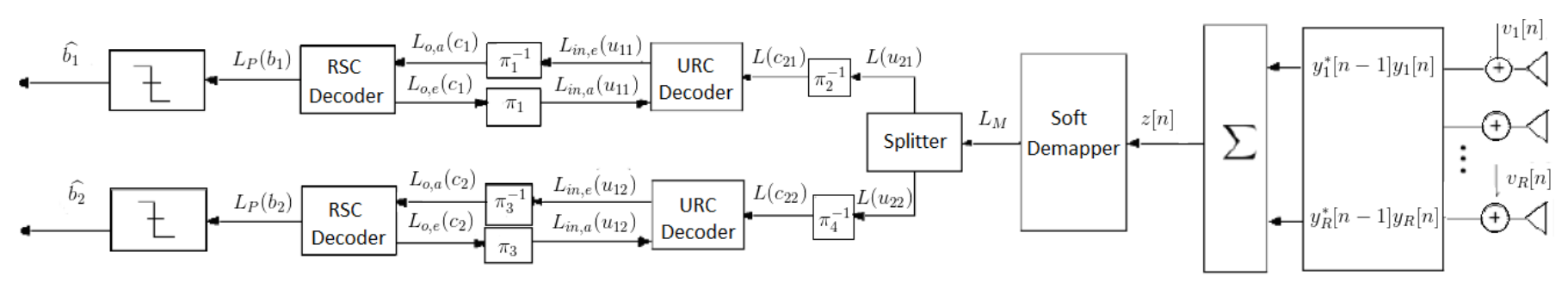

Fig. 2. Receiver of a Non Coherent m-SIMO system with coding for $P=2$ users.

[5] and (5), we can express the SINR as a function of $\rho$ and $R$ as follows

$$
\operatorname{SINR}=\frac{R}{P} \frac{\rho^{2}}{\rho^{2}+2 \rho+1} .
$$

Then, the demapper's extrinsic information transfer for our particular m-SIMO system depends both on the apriori information and on the SINR, which is formulated as

$$
L_{i n, e}(\mathbf{u})=T\left[L_{i n, a}(\mathbf{u}), \operatorname{SINR}\right]=T\left[L_{i n, a}(\mathbf{u}), R, \rho\right],
$$

For the outer decoder, we employ a set of 17 convolutional codes defined in [9], whose coding rate spans the range [1/10-9/10]. We select the minimum $R$ value for ensuring an open EXIT tunnel with one of the 17 sub-codes, ensuring an infinitesimally low BER.

\section{B. Channel Model}

In order to study the behavior of the system in time-varying channels, we normalize the channel's bandwidth $(B W)$ by the Doppler shift $\left(f_{D}\right)$ to define a metric that relates both parameters as

$$
\beta=\frac{B W}{f_{D}}
$$

In order to consider this temporal correlation encapsulated in $h_{i j}$ for our simulations, we generate complex Gaussian noise in the frequency domain, which is filtered using a Doppler filter characterize by a bandwidth of $f_{D}$.

The advantage of using this metric is that it allows us to characterize the system independently of the physical system parameters such as the carrier frequency $\left(f_{c}\right)$, bandwidth or Doppler effect. Thus, it is a beneficial metric for designing new systems for practical time-variant wireless channels. This model can be applied both for conventional frequencies and also for $\mathrm{mm}$-wave or $\mathrm{THz}$ frequencies [7].

\section{ANALysis of the ReQuired Number of ANTENNAS FOR BER CONVERGENCE}

In this section, we analyze the effect of time-varying channels on the attainable performance of our non-coherent system based on DPSK and BICM-ID using a high number of antennas. The first objective of this analysis is to find the minimum value of $R$ required to attain a specific target performance, such as a BER of $10^{-4}$ or below, when considering a timevarying channel. We also seek to get the value of $\beta$, where the effect of the channel's time variation becomes negligible in terms of its effect on the performance of NC detection.
Upun using the EXIT chart analysis detailed in [5], we list in Table I the number of antennas $R$ required for attaining an open EXIT tunnel, when considering a constant channel and also for different degrees of channel variability $\beta$. These $R$ values sometimes require a high number of iterations, when the open EXIT tunnel is narrow. As $\beta$ decreases, which results in more violent channel time variation, the $R$ required for achieving the same performance as that of the constant channel increases. We can maintain the same $R$ as for the constant channel at the expense of a reduced throughput by using a lower channel coding rate. When the coding rate is low, increasing the reference SNR $\rho$, makes the effect of time variations less visible. This is not, however, the case for high coding rates. Also, for $\rho=0 \mathrm{~dB}$ and $\beta=3$ there are certain coding rates that do not have an open tunnel for any $R$. For instance, in order to decode a $1 / 2$-rate RSC code we have to increase $R$ from 90 to 180 antennas, when we have a channel characterized by $\beta=3$ or to 110 antennas for the case $\beta=5$, for keeping the same performance as the constant channel, as shown in Table I. In Fig. 3, we show the EXIT-curve for $\beta=3$ and 5 in conjunction with their decoding trajectories, where the energy efficiency attained by our m-SIMO is clearly evidenced by the fact that a low $\rho$ is sufficient for having an open EXIT tunnel.

The EXIT chart of the system associated with $\rho=0 \mathrm{~dB}$ and $\beta$ values ranging from 1.5 to 6 by steps of 0.5 is shown in Fig. 4 and Fig. 5 for $R=100$ and 50 antennas, respectively. The effect of $\beta$ is shown in both figures for values lower than 10 . The system exhibits a similar performance to the constant channel for $\beta \geq 10$. Hence, we note that our system is capable of withstanding $f_{D}$ close to the channel's bandwidth as (10). We note that for $\beta>6$ we can select a $1 / 2$-rate RSC for $R=100$ antennas and a $1 / 4-$ rate RSC for $R=50$ antennas. However, a large number of iterations is required for ensuring that the performance becomes equivalent to that of the constant-envelope channel, since the EXIT-tunnel is narrower than for $\beta \geq 10$.

In Fig. 6 we show the BER for 2 users, $\rho=0 \mathrm{~dB}, M=4$. In this case, we evaluate the effect of the time varying channel on the BER performance. We observe having an infinitesimally low BER at $R=140$ antennas for $\beta=5$ and $R=300$ antennas for $\beta=3$, requiring an increase of 50 and 200 antennas, respectively, with respect to the constant-envelope channel. This result matches with the EXIT chart predictions in Fig. 3.

Finally, we examine the typical value of $\beta$ in the current wireless standards. In LTE, where we have $f_{c}=2.6 \mathrm{GHz}$, 
TABLE I

PERFORMANCE FOR $P=2$ USERS, EEP CONSTELLATION FOR DIFFERENT RSC ENCODERS AND $\beta$.

\begin{tabular}{|c|c|c|c|c|c|c|c|c|c|c|}
\hline & \multirow[t]{2}{*}{$\begin{array}{l}\text { Outer } \\
\text { RSC }\end{array}$} & \multicolumn{3}{|c|}{$\begin{array}{l}\text { Required } R \text { for each SNR: } \\
\text { Constant Channel }\end{array}$} & \multicolumn{3}{|c|}{$\begin{array}{l}\text { Required } R \text { for each SNR: } \\
\qquad \beta=3\end{array}$} & \multicolumn{3}{|c|}{$\begin{array}{l}\text { Required } R \text { for each SNR } \\
\qquad \beta=5\end{array}$} \\
\hline & & $0 \mathrm{~dB}$ & $3 \mathrm{~dB}$ & $6 \mathrm{~dB}$ & $0 \mathrm{~dB}$ & $3 \mathrm{~dB}$ & $6 \mathrm{~dB}$ & $0 \mathrm{~dB}$ & $3 \mathrm{~dB}$ & $6 \mathrm{~dB}$ \\
\hline \multirow{17}{*}{ 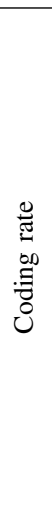 } & $1 / 10$ & 20 & 20 & 20 & 40 & 25 & 20 & 25 & 20 & 20 \\
\hline & $3 / 20$ & 30 & 20 & 20 & 60 & 35 & 25 & 35 & 20 & 20 \\
\hline & $1 / 5$ & 40 & 20 & 20 & 70 & 40 & 30 & 45 & 25 & 20 \\
\hline & $1 / 4$ & 50 & 90 & 20 & 90 & 50 & 35 & 55 & 35 & 25 \\
\hline & $3 / 10$ & 55 & 30 & 20 & 100 & 60 & 35 & 65 & 40 & 25 \\
\hline & $7 / 20$ & 60 & 35 & 25 & 120 & 65 & 45 & 75 & 45 & 30 \\
\hline & $2 / 5$ & 70 & 40 & 30 & 140 & 80 & 55 & 85 & 50 & 35 \\
\hline & $9 / 20$ & 80 & 45 & 30 & 150 & 90 & 65 & 95 & 55 & 40 \\
\hline & $1 / 2$ & 90 & 50 & 35 & 180 & 105 & 75 & 110 & 60 & 45 \\
\hline & $11 / 20$ & 100 & 60 & 40 & 210 & 120 & 85 & 120 & 70 & 50 \\
\hline & $3 / 5$ & 120 & 65 & 45 & 240 & 145 & 100 & 140 & 80 & 60 \\
\hline & $13 / 20$ & 130 & 75 & 55 & 280 & 170 & 120 & 160 & 90 & 70 \\
\hline & $7 / 10$ & 150 & 90 & 60 & 320 & 200 & 140 & 180 & 105 & 80 \\
\hline & $3 / 4$ & 180 & 100 & 70 & 450 & 240 & 180 & 210 & 120 & 90 \\
\hline & $4 / 5$ & 210 & 120 & 85 & 500 & 300 & 210 & 250 & 140 & 100 \\
\hline & $17 / 20$ & 260 & 150 & 100 & $\mathrm{X}$ & 400 & 270 & 350 & 170 & 120 \\
\hline & $9 / 10$ & 350 & 180 & 130 & $\mathrm{X}$ & 500 & 310 & 360 & 210 & 150 \\
\hline
\end{tabular}

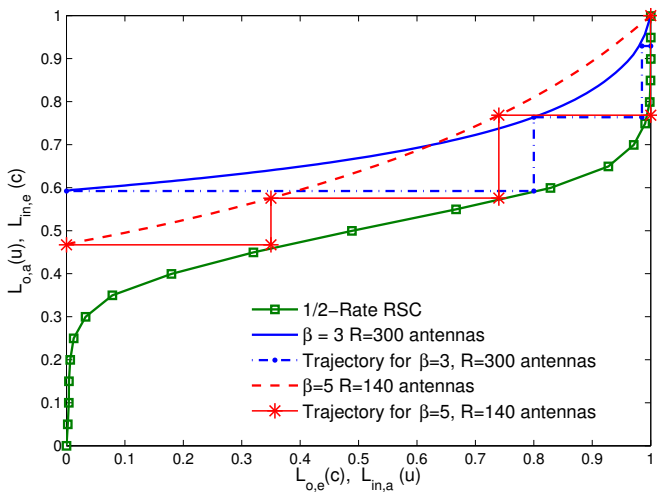

Fig. 3. EXIT Chart for $P=2$ users, $\rho=0 \mathrm{~dB}, M=4, \beta=3$ and $\beta=5$.

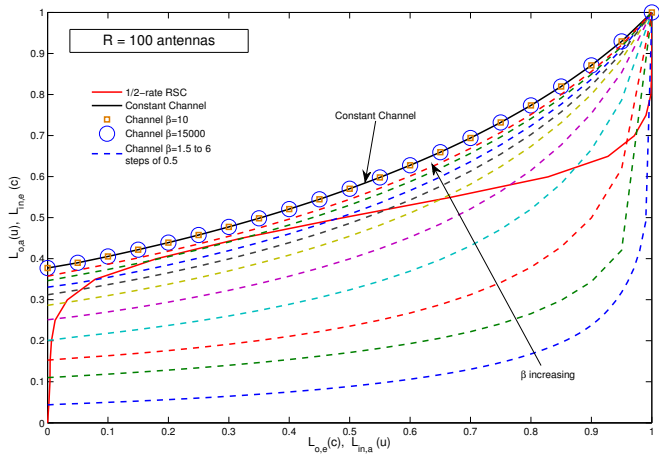

Fig. 4. EXIT Chart for $P=2$ users, $\rho=0 \mathrm{~dB}, M=4, \beta$ from 1.5 to 6 by steps of 0.5 . For $R=100$ antennas.

a $B W$ of $20 \mathrm{MHz}$ and a mobile velocity of $v=120 \mathrm{Km} / \mathrm{h}$, we get a value of $f_{D}=v / \lambda=290 \mathrm{~Hz}$. Then, according to (10) we have $\beta=68,970$. Additionally, when we have $f_{D}=200 \mathrm{~Hz}$ and $v=83 \mathrm{Km} / \mathrm{h}$, we arrive at $\beta=10^{5}$. In both cases $\beta$ is very high, ensuring that we achieve the same performance as in the constant-envelope channel scenario. On

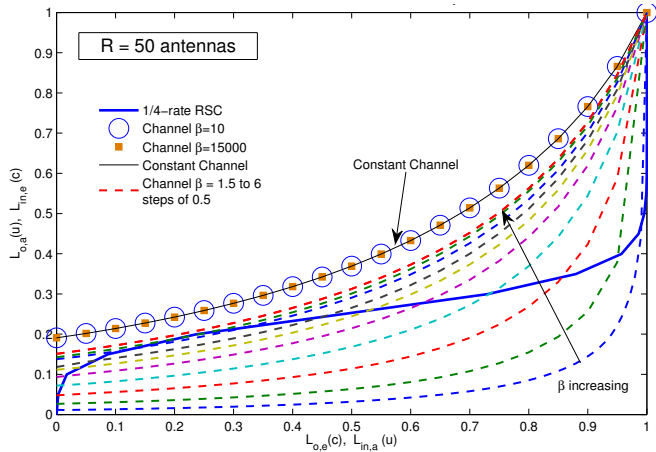

Fig. 5. EXIT Chart for $P=2$ users, $\rho=0 \mathrm{~dB}, M=4, \beta$ from 1.5 to 6 by steps of 0.5 . For $R=50$ antennas.

the other hand, for mm-wave links, due to the high $f_{c}$, the Doppler shift is also high. For instance, employing $B W=100$ $\mathrm{MHz}$ at $f_{c}=60 \mathrm{GHz}$, we have $f_{D}=6.7 \mathrm{KHz}$ and considering $v=120 \mathrm{Km} / \mathrm{h}$, we get $\beta=15,000$. With this value we have again the same performance as for the constant-envelope channel scenario. Furthermore, if we consider LTE for high speed railway systems characterized by a high Doppler spread or short coherence time $T_{c}$ with $v=500 \mathrm{Km} / \mathrm{h}$, we have a $f_{D}=1.2 \mathrm{KHz}$ and $\beta=16,615$, which again guarantees the same performance as for the constant-envelope channel. With these examples, we can deduce that our system is very robust to the temporal variations.

\section{Achievable Bit Rate}

In this section, we characterize the maximum achievable rate (MAR) of our non-coherent m-SIMO-DPSK-BICM-ID scheme. The EXIT chart can be used to evaluate the MAR as discussed in [9], which indicates that the MAR can be obtained using the area $A$ under the EXIT curve of the inner code. In our system, the inner code is the URC code shown in Fig. 2. Here, we translate the expression in [9] to evaluate 


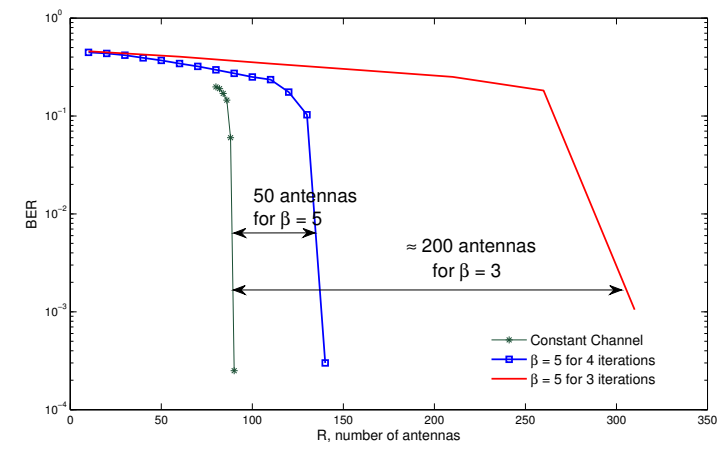

Fig. 6. BER for $P=2$ users, $\rho=0 \mathrm{~dB}, M=4$, URC, EEP and $\beta=3$ and $\beta=5$

the MAR as a function of the number of antennas $R$, since we parametrize the EXIT curve as a function of $R$. The area $A$ is calculated for different $R$ values, denoted as $A_{R}$, then the maximum achievable rate $\eta_{\max }$ may be defined in terms of $R$ :

$$
\eta_{\max }(R)=\log _{2}(M) \cdot A_{R}
$$

Then, in order to obtain the MAR of (11) that our system can support, for each $\rho, R$ and a candidate constellation we chose the EXIT curve that has an open tunnel. If there is indeed an EXIT chart that has an open tunnel, this means that this modulation order $M$ is decodable with a vanishingly low BER. In order to keep a feasible value of $R$, we select the modulation order from the set $\mathfrak{M}=\{2,4,8,16\}$, since higher values of $M$ require a significantly higher number of antennas. Then, the maximum achievable rate is given by the maximum modulation order that we found to be decodable multiplied by the coding rate as follows:

$$
\eta_{p}\left(R, \beta, M_{i}\right)=\log _{2}\left(M_{i}\right) \cdot \text { rate }
$$

where rate is the original outer code rate.

For a given $\rho$ and $R$ value, we can draw an EXIT chart for each possible value of the constellation order $M_{i}$. With the aid of each of these EXIT charts we can select the coding scheme that produces an open tunnel at the lowest value of the outer code rate.

In Fig. 7 we show the achievable rate of the m-SIMODPSK-BICM-D system, when $R=100$ antennas, as a function of the SNR $\rho$. In Fig. 7, we show the constellation order $M_{i} \in \mathfrak{M}$ that provides this lower outer code rate value and the achieved $\eta$ according to (15). The dotted line in Fig. 7 is the MAR provided by the EXIT chart and represents the upper bound of MAR for our system. The solid lines are obtained for different $\beta$ values. We note that the difference between the theoretical MAR and the practically achieved MAR is small for $\beta \geq 10$, while for smaller $\beta$ there is a noticeable degradation.

\section{Conclusions}

In this paper, we have analyzed our non-coherent massive SIMO system relying on M-DPSK and BICM-ID operating in time-varying channels. We have used a metric to model the time-varying characteristics of the channel so that the

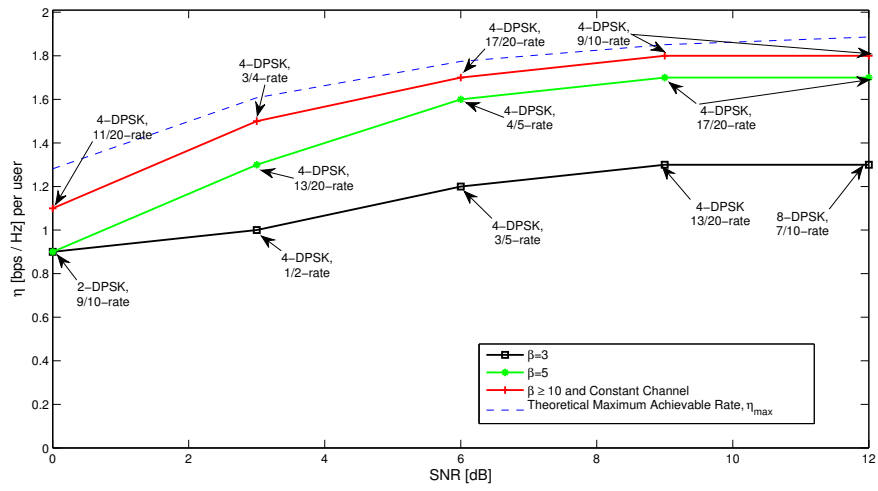

Fig. 7. Maximum Achievable Rate for $R=100$ antennas.

analysis can be applied to any wireless communication standard. We have employed EXIT charts as a powerful technique of analyzing and designing iteratively decoded systems. The analysis based on EXIT chart allows us to obtain an estimate of the degradation of the system's performance imposed by realistic channels. Hence, we have shown that our proposed system is robust to temporal variations. On the other hand, we have shown that the practically achievable rate relying on the modulation and coding schemes considered approaches the theoretically achievable rate of M-DPSK combined with BICM-ID.

\section{ACKNOWLEDGMENT}

This work has been partially funded by the MIMOTEX (TEC2014-61776-EXP) and the Spanish national project ELISA (TEC2014-59255-C3-3-R).

\section{REFERENCES}

[1] E. Bjornson, E. G. Larsson, and T. L. Marzetta, "Massive MIMO: ten myths and one critical question," IEEE Communication Magazine, vol. 54, no. 2, pp. 114-123, 2016.

[2] D. Qiao, Y. Wu, and Y. Chen, "Massive MIMO architecture for 5G networks: Co-located, or distributed?," in Proc. 11th ISWCS, Barcelona, Spain, pp. 192-197, Aug. 2014

[3] J. Zhang, B. Zhang, S. Chen, X. Mu, M. El-Hajjar, and L. Hanzo, "Pilot contamination elimination for large-scale multiple-antenna aided OFDM systems," IEEE Journal of Selected Topics in Signal Processing, 8, (5), pp. 759-772, 2014.

[4] Alexandros Manolakos, Mainak Chowdhury, Andrea Goldsmith, "Energy-based Modulation for Noncoherent Massive SIMO Systems," IEEE Transactions on Wireless Communications, 2016

[5] V. M. Baeza, A. G. Armada, W. Zhang, M. El-Hajjar and L. Hanzo, "A Non-Coherent Multi-User Large Scale SIMO System Relying on M-ary DPSK and BICM-ID," submitted to IEEE TVT, available at http://www.tsc.uc3m.es/ agarcia/tmp/TVT-R1.pdf, 2016.

[6] X. Li and J. A. Ritcey, "Bit-Interleaved Coded Modulation with Iterative Decoding," IEEE Commun. Letters, vol. 1, pp. 169-171, Nov. 1997.

[7] T. S. Rappaport, S. Sun, R. Mayzus, H. Zhao, Y. Azar, K. Wang, G. N. Wong, J. K. Schulz, M. Samimi, and F. Gutierrez, "Millimeter Wave Mobile Communications for 5G Cellular: It Will Work!," IEEE Access, vol. 1, pp. 335-349, May 2013.

[8] T. Krner and S. Priebe, "Towards thz communications-status in research, standardization and regulation," Jounal of Infrared, Milimeter and Terahertz Waves, vol. 35, no. 1, pp. 547-558, 2014.

[9] M. El-Hajjar and L. Hanzo, "EXIT Charts for System Design and Analysis," IEEE CST, vol. 99 pp. 1-27, May 2013. 